(2) Open Access Full Text Article

\title{
Comparison of $0.2 \%$ and $0.18 \%$ hyaluronate eye drops in patients with moderate to severe dry eye with keratitis or keratoconjunctivitis
}

This article was published in the following Dove Press journal:

Clinical Ophthalmology

6 April 2017

Number of times this article has been viewed

\author{
Dorothea Groß' \\ Marc Childs ${ }^{2}$ \\ Jean-Marie Piaton ${ }^{3}$ \\ 'Ursapharm Arzneimittel GmbH, \\ Saarbrücken, Germany; ${ }^{2}$ Laboratoires \\ Delbert, Paris, ${ }^{3}$ Ophthalmic Practice, \\ Domont, France
}

Purpose: Comparison of efficacy and safety of $0.2 \%$ and $0.18 \%$ hyaluronic acid (HA) eye drops three times a day (tid) in patients with moderate to severe dry eye disease, related to keratitis or keratoconjunctivitis.

Patients and methods: Prospective, multicenter, randomized, single-masked, phase IIIb, noninferiority study $(0.2 \% \mathrm{HA}$ vs $0.18 \% \mathrm{HA})$ in two parallel groups over a period of 84 days. $\mathrm{N}=70$ patients were evaluated. Primary efficacy outcome was ocular surface (OS) staining change on day 35 (D35), compared to baseline. Fluorescein and lissamine green were used for staining of cornea and conjunctiva. Secondary efficacy outcome included tear film breakup time, OS staining score on day 84 (D84), ocular comfort index, as well as patients' and doctors' evaluation.

Results: Compared to day 0 (D0), $0.2 \%$ HA achieved a $47.7 \%$ reduction in staining score $(-3.00 \pm 2.81$ [standard deviation] points, $n=38$ patients) at $\mathrm{D} 35 ; 0.18 \%$ HA showed a $41.2 \%$ reduction $(-2.59 \pm 2.20$ [standard deviation] points, $n=32$ patients). Statistical analysis showed noninferiority in efficacy of $0.2 \% \mathrm{HA}$ compared to $0.18 \% \mathrm{HA}$ on D35. At D84, the reduction in staining score had further increased to $64.5 \%$ for $0.2 \%$ HA and to $56.4 \%$ for $0.18 \%$ HA. Both eye drops improved tear film breakup time and ocular comfort index values. Investigators and patients assessed both treatments with 5 of 7 points (Likert Scale, medians). The rate of adverse events (AE) was $2.3 \%$ for $0.2 \% \mathrm{HA}$ and $7.1 \%$ for $0.18 \% \mathrm{HA}$ with no serious AE.

Conclusion: $0.2 \%$ and $0.18 \%$ HA eye drops significantly improved signs and symptoms of dry eye disease and were well tolerated with few AEs. Noninferiority of $0.2 \%$ HA compared to $0.18 \%$ HA was demonstrated for reduction of OS lesions. In some parameters, there was a nonsignificant trend in favor of $0.2 \%$ HA concentration.

Keywords: dry eye, hyaluronic acid, ocular surface lesions, ocular staining, keratoconjunctivitis sicca, eye drops, unpreserved, hyaluronate, fluorescein, lissamine green

\section{Introduction}

Dry eye disease (DED) is a multifactorial condition that affects tears and ocular surface (OS). ${ }^{1,2}$ DED can be associated with inflammation. ${ }^{3}$ Lesions of the cornea and conjunctiva may occur, affecting the integrity of the OS. ${ }^{1}$ Patients with DED may experience foreign body sensation, burning, itching and aching sensations, heavy eyes, fatigued eyes, and a decreased quality of vision, eg, stray light. ${ }^{4}$ DED may also increase the risk of infections of the OS. ${ }^{5}$ The current understanding of the pathogenesis of dry eye is that the instability of the tear film and the increase of osmolarity result in a vicious circle and lead to inflammation of the OS. ${ }^{3}$

Substitution therapy is the therapy of choice for most forms of DED. The goal of topical therapy in the form of lubricating eye drops is to control the activity and
Correspondence: Dorothea Groß Ursapharm Arzneimittel GmbH, Industriestrasse 35, D-66I29 Saarbrücken, Germany

Tel +4968059292132 Fax +496805929287

Email dorothea.gross@ursapharm.de (c) hereby accept the Terms. Non-commercial uses of the work are permitted without any further permission from Dove Medical Press Limited, provided the work is properly attributed. For permission for commercial use of this work, please see paragraphs 4.2 and 5 of our Terms (https://www.dovepress.com/terms.php). 
progression of the disease, to decrease signs and symptoms related to dry eye, and, as such, to contribute to prevent or delay health consequences. ${ }^{2}$

Eye drops for DED therapy usually contain chemically inert macromolecules that adhere to the OS and can bind and retain water molecules. One of today's most commonly used polymers for tear substitution is sodium hyaluronate, resp. hyaluronic acid (HA). HA is a naturally occurring glycosaminoglycan or mucopolysachharide with a molecular weight of 50,000-8,000,000 Da depending on substrate and method of presentation. HA is considered the most recent generation of dry eye lubricating therapy and is a naturally occurring polysaccharide mainly found in the vitreous body of the eye ${ }^{6}$ as well as in tear fluid. HA has been used for many years in ophthalmology as a viscoelastic during cataract surgery, keratoplasty, and other procedures. HA eye drops for DED treatment entered the market in the early $1990 \mathrm{~s}^{7}$ Concentrations of HA in ophthalmics range from $0.003 \%$ to $0.4 \%{ }^{8,9}$ For DED therapy, higher HA concentrations, between $0.1 \%$ and $0.4 \%$, are usually used. . $^{70-15}$

Preservative-free artificial tears have become more and more common in DED therapy. This is due to the fact that preservatives in ophthalmics, including the most frequently used benzalkonium chloride, have cytotoxic properties, disturbing the tear film. Especially during long-term use, preserved eye drops may cause more damage than benefit. When added as excipients in eye drops, preservatives may counteract the beneficial effect of the other ingredients and may even cause DED. ${ }^{16}$

In previous clinical investigations performed in a similar population, $0.18 \%$ HA was demonstrated to be statistically significantly superior on the relief of symptoms of dry eyes over a saline solution and as noninferior to a $0.3 \%$ carbomer solution in a 304 patients, three parallel group clinical study. ${ }^{11}$ Based on these findings, the choice of $0.18 \%$ HA as comparator for a $0.2 \%$ HA formulation appeared to be scientifically relevant to perform this noninferiority study.

Some of the most serious consequences of untreated DED are lesions or epithelial defects of the cornea and conjunctiva. Cell defects can be quantified by staining with fluorescein or lissamine green. ${ }^{17}$ While fluorescein stains corneal lesions by permeating in intercellular spaces associated with epithelial cellular disruption, ${ }^{18}$ lissamine green stains damaged or devitalized conjunctival cells. ${ }^{19}$ Our study used staining scores to quantify the clinical effect of unpreserved 0.2\% HA (Hylo ${ }^{\circledR}$ Confort Plus; Laboratoires Ursapharm Sas, Sarreguemines, France), which has a molecular weight of 2,400,000-2,800,000 Da and 0.18\% HA (Vismed ${ }^{\circledR}$ Multi, TRB Chemedica AG, Haar, Germany). The primary goal of this study was to test noninferiority of efficacy of the preparation of $0.2 \%$ HA compared to $0.18 \%$ HA.

This comparison was made in a setting with patients with moderate to severe DED related to keratitis or keratoconjunctivitis. The primary outcome parameter was a composite staining score of cornea and conjunctiva. Moreover, the study examined the change of the ocular comfort index (OCI) in the course of the therapy with $0.2 \% \mathrm{HA}$ and $0.18 \%$ HA. Patients' and investigators' treatment satisfaction was also assessed.

\section{Patients and methods}

\section{Participants}

The per-protocol (PP) population consisted of 70 patients with moderate to severe dry eye and noninfectious, nonviral keratitis, or keratoconjunctivitis. Thirty-eight patients were included in the $0.18 \%$ HA group, and 32 in the $0.2 \%$ HA group.

For inclusion, patients had to fulfill all of the following 6 criteria: 1) age $>18$ years with moderate to severe form of dry eyes with keratitis or keratoconjunctivitis; 2) use of lubricants in the previous months and despite this treatment presenting symptoms of keratoconjunctivitis and corneal or conjunctival lesions (objectified by OS staining); 3 ) presenting at least 3 among 6 dry eye symptoms (frequency score $>1$ and intensity score $>2$ on the OCI related to moderate to severe dry eye syndrome; 4) at least on the same eye these 2 conditions: a) overall staining score of the OS between $>4$ and $<9$ according to the 15 points Oxford Scale (assessing the diagnosis of keratoconjunctivitis by OS staining), b) at least one of the following objective signs of decreased tear volume: unanesthetized Schirmer test between $3 \mathrm{~mm}$ and $9 \mathrm{~mm}$ within $5 \mathrm{~min}$ or sum of 3 measures of tear break up time (TBUT) <30s; 5) patient willing and able to comply with the protocol requirements; and 6) collection of written informed consent, signed and dated.

Exclusion criteria were patient unable to comply with study requirements; previous participation to this study or if the patient was the investigator or one of his/her team member; participation in another clinical study in the last 4 weeks preceding or following the end of this study; best-corrected visual acuity $<1 / 10$; moderate or severe blepharitis; dry eye related to the following: eyelid abnormality, corneal disease not related to DED, OS metaplasy, filamentary keratitis, corneal neovascularization; ocular trauma; ocular infection, or ocular inflammation $<3$ months; ocular allergy or ocular herpetic $<3$ months; infectious or viral acute ocular pathology; history of inflammatory corneal ulcer, recurrent erosion, or uveitis; cataract surgery, in situ keratomileusis laser, photoreactive keratectomy, other photorefractive 
surgery, and other surgery of the limbus or corneal incision in the previous 12 months; treatment with isotretinoïd, cyclosporine, tacrolimus, sirolimus, pimecrolimus, punctal plugs or cauterization, hormonal treatment, or topical betablockers; use of ophthalmic treatment in the last 2 weeks, except lubricating tears; any OS disease did not related to dry eyes; repeated alcohol consumption or abuse of illegal drugs; pregnancy or breast-feeding women known at the inclusion; women of childbearing potential who did not use an efficacious contraceptive method (estroprogestative, intrauterine device) and in the absence, performance of a pregnancy test, the result being obtained before the fluorescein staining realized in the context of the study.

\section{Study design}

This was a multicentre, phase IIIb, single-blind (the investigator was kept unaware of the investigational product allocated at randomization by a pseudonymized patient unit packaging), randomized study in two parallel groups. This clinical trial was registered under the French ANSM registry number: RCB 2014-A01812-45.

The protocol complied with recommendations of the 18th World Health Congress (Helsinki, 1964) and all applicable amendments laid down by the World Medical Association and the ICH guidelines for Good Clinical Practice. The protocol also complied with all international and national laws and regulations, in which the clinical trial was performed, as well as any applicable guidelines. Written informed consent was received from all participants for this study, the protocol, and all other appropriate study-related documents were submitted by the investigator for review and written ethical approval was received from the appropriate independent ethics committee and institutional review board: Comité de Protection des Personnes Ile-de-France VIII, Hôpital Ambroise Paré, Paris.

The study was performed as a multicentric study at 15 centers (of which 12 were active) in France.

The trial was conducted in two phases: day 0 (D0) to day 35 (D35) phase to compare the relative efficacy of both investigational products on the main efficacy criteria. The D35 to D84 follow-up phase served to evaluate the efficacy and the safety of each product. The treatment was continued masked with the investigational product allocated at D0.

\section{Prior and concomitant therapy}

Any topical ocular ointment or eye drops were forbidden during the study. Systemic treatments were allowed with the exception of hormonal treatments, isotretinoïds, cyclosporine, tacrolimus, sirolimus, or pimecrolimus.

\section{Study treatment}

Investigational treatment: $0.2 \%(2 \mathrm{mg} / \mathrm{mL}) \mathrm{HA}$ sterile solution for topical ophthalmic use (Hylo Confort Plus/Hylo Gel), preservative-free solution in a bottle of $10 \mathrm{~mL}$.

Comparator treatment: $0.18 \%(1.8 \mathrm{mg} / \mathrm{mL})$ HA sterile solution for topical ophthalmic use (Vismed ${ }^{\circledR}$ Multi), preservative-free solution in a multidose bottle of $10 \mathrm{~mL}$. Dosing for both products was one drop three times a day (tid) in the affected eye(s).

\section{Patient visits}

Patients presenting the diagnosis of DED were evaluated by the OCI, OS staining (15-point Oxford Scale), TBUT, and Schirmer test. When respecting the inclusion and exclusion criteria, they were recruited into the study at Visit 1 (baseline, D0). At Visit 2 (D35 \pm 4 days), the patient returned for a follow-up consultation. For efficacy and safety, patients were asked to fulfill the symptoms questionnaire (OCI), underwent ophthalmological tests, and a comprehensive clinical interview for safety as well as for satisfaction. At Visit 3 (D84 \pm 7 days), frequency and intensity of symptoms (OCI), ocular staining, and other ophthalmological tests were reassessed. Compliance was evaluated by counting returned bottles by the patients.

\section{Scales}

Fifteen points Oxford Scale ${ }^{17}$ : the amount of OS lesions at the cornea, and nasal or temporal conjunctival zones, obtained by fluorescein (staining corneal lesions) and lissamine green (staining damaged or devitalized conjunctival cells) staining was assessed. Oxford Scale subscores were cornea (5 points), nasal (5 points), or temporal (5 points) conjunctival zones at D35 and D84. For the most affected eye at D0, the difference (D35-D0) was evaluated.

OCI: Patients were asked to report on a semiquantitative 0-6 points scale the frequency and the intensity of the six following items occurring in the previous week: dryness, grittiness, stinginess, fatigue, pain, and itching $(0=$ absent, $6=$ maximum).

Efficacy according to the investigator and patients' satisfaction were reported on the 7-level qualitative Likert scale at D35 and D84, described in the literature in detail. ${ }^{20}$

\section{Statistical analysis}

Data of one eye per patient (the most affected eye) were used for statistical analysis. All statistical analyses were performed at a probability threshold of 5\% for significance using twosided tests or two-sided confidence intervals. All summaries and statistical analyses were generated using SAS, version 9.4 
(SAS Institute Inc., Cary, NC, USA). PP population has been used as primary analysis and included all patients from the intent-to-treat (ITT) population without any major protocol violation.

Primary efficacy outcome was the change of Oxford staining score after 35 days of treatment (D0-D35). The hypothesis to conclude a noninferiority of $0.2 \% \mathrm{HA}$ vs $0.18 \%$ HA was determined as a difference on the overall score of the Oxford Scale $<2$ points.

Secondary efficacy outcome included: OS staining score on D84, TBUT, 6-item OCI index in frequency and intensity, and patients' and investigators' evaluation of treatment.

\section{Results}

\section{Patient demographics}

Patients' mean age was $59.7 \pm 15.4$ years (mean \pm standard deviation [SD]). Fifty-nine (69.4\%) patients were female and $26(30.6 \%)$ were male. Baseline patient characteristics are shown in Table 1.

\section{Patient history of dry eye}

Patients receiving $0.2 \% \mathrm{HA}$ had a diagnostic history of dry eye for $6.81 \pm 4.62$ years (mean $\pm \mathrm{SD}$ ), whereas patients receiving $0.18 \%$ HA had a history of $5.08 \pm 3.57$ years (mean $\pm \mathrm{SD}$ ) with no significant difference between groups $(P<0.0988$, Mann-Whitney $U$-test $)$.

\section{Triggering factors for dry eye}

Patients were asked for triggering factors including pollution, smoking, wind, work on screen, contact lenses, or chronic treatment known to be related to dry eye such as treatments

Table I Demographic data at baseline (per-protocol population)

\begin{tabular}{|c|c|c|}
\hline $\begin{array}{l}\text { Background } \\
\text { factors }\end{array}$ & $\begin{array}{l}0.2 \% \text { HA } \\
N=43\end{array}$ & $\begin{array}{l}0.18 \% \mathrm{HA} \\
\mathrm{N}=42\end{array}$ \\
\hline \multicolumn{3}{|l|}{ Gender } \\
\hline Female & 34 (79.1\%) & 25 (59.5\%) \\
\hline Male & $9(20.9 \%)$ & 17 (40.5\%) \\
\hline \multicolumn{3}{|l|}{ Age (years) } \\
\hline Mean & $62.09(15.64)$ & $57.26(15.00)$ \\
\hline Median (range) & $63(26-85)$ & $60(24-88)$ \\
\hline \multicolumn{3}{|l|}{ Weight (kg) } \\
\hline Mean (SD) & $66.44(13.85)$ & $70.52(10.61)$ \\
\hline Median (range) & 66 (45-98) & $70(5 \mid-98)$ \\
\hline \multicolumn{3}{|l|}{ Height (cm) } \\
\hline Mean (SD) & $163.56(7.45)$ & I69.2I (7.30) \\
\hline Median (range) & $163(146-185)$ & $170(158-185)$ \\
\hline \multicolumn{3}{|l|}{ BMI $\left(\mathrm{kg} / \mathrm{m}^{2}\right)$} \\
\hline Mean (SD) & $24.86(5.04)$ & 24.61 (3.30) \\
\hline Median (range) & $23.2(16.4-40.3)$ & $24(19.1-33.9)$ \\
\hline
\end{tabular}

Abbreviations: BMI, body mass index; HA, hyaluronic acid; SD, standard deviation.
Table 2 Number of DED triggering factors

\begin{tabular}{llllll}
\hline $\begin{array}{lllll}\text { Number of } \\
\text { triggering factors }\end{array}$ & \multicolumn{2}{l}{ Group } \\
\cline { 2 - 3 } & $\mathbf{0 . 2 \% ~ H A ~}$ & & \multicolumn{2}{l}{$\mathbf{0 . 1 8 \% ~ H A ~}$} \\
\cline { 2 - 3 } \cline { 5 - 6 } & $\mathbf{N}$ & $\%$ & & $\mathbf{N}$ & $\%$ \\
\hline 1 & 18 & 47.4 & & 12 & 37.5 \\
2 & 12 & 31.6 & & 17 & 53.1 \\
3 & 8 & 21.1 & & 9.4 \\
Total & 38 & 100.0 & & 32 & 100.0 \\
\hline
\end{tabular}

Abbreviations: DED, dry eye disease; HA, hyaluronic acid.

for anxiety, menopause, Parkinson's disease, orinsomnia. In both groups, most patients had 1 or 2 triggering factors (Table 2).

\section{Treatments in the past}

In both groups, most patients had 1 topical treatment of DED with lubricating medications before this study (Table 3).

\section{Efficacy outcomes}

Primary efficacy

Data are presented for the most affected eye. Baseline staining at D0 of OS lesions was 6.28 for $0.18 \%$ HA ( $n=38$ patients) and 6.29 for $0.2 \%$ HA ( $n=32$ patients) on the 15-point Oxford Scale. Figure 1 shows results of D0, D35, and D84. On D35, patients receiving 0.2\% HA showed a $47.7 \%$ reduction in staining score points $(-3.00 \pm 2.81$, mean $\pm \mathrm{SD}$ ) and patients with $0.18 \%$ HA showed a $41.2 \%$ reduction $(-2.59 \pm 2.20)$.

The OSC difference between the 2 HA concentrations on D35 is -0.41 and in favor of $0.2 \%$ HA. The $95 \%$ confidence interval is from -1.6265 to the upper limit at 0.8140 . As this upper limit of the $95 \%$ confidence interval is $<2$, it was demonstrated that on this primary efficacy endpoint, $0.2 \%$ HA is noninferior to $0.18 \%$ HA $(P<0.0001)$.

\section{Secondary efficacy}

At D84, the overall score of the Oxford Scale indicated improvement in both treatment groups. The difference to D0

Table 3 Number of topical DED medications in the past

\begin{tabular}{|c|c|c|c|c|}
\hline \multirow{3}{*}{$\begin{array}{l}\text { Number of } \\
\text { treatments } \\
\text { for dry eye } \\
\text { in the past }\end{array}$} & \multicolumn{4}{|c|}{ Group } \\
\hline & \multicolumn{2}{|c|}{$0.2 \%$ HA } & \multicolumn{2}{|c|}{$0.18 \%$ HA } \\
\hline & $\bar{n}$ & $\%$ & $n$ & $\%$ \\
\hline I & 18 & 47.4 & 21 & 65.6 \\
\hline 2 & II & 28.9 & 8 & 25.0 \\
\hline 3 & 6 & 15.8 & 2 & 6.3 \\
\hline 4 & 1 & 2.6 & 0 & 0 \\
\hline 5 & 2 & 5.3 & I & 3.1 \\
\hline Total & 38 & 100.0 & 32 & 100.0 \\
\hline
\end{tabular}

Abbreviations: DED, dry eye disease; HA, hyaluronic acid. 


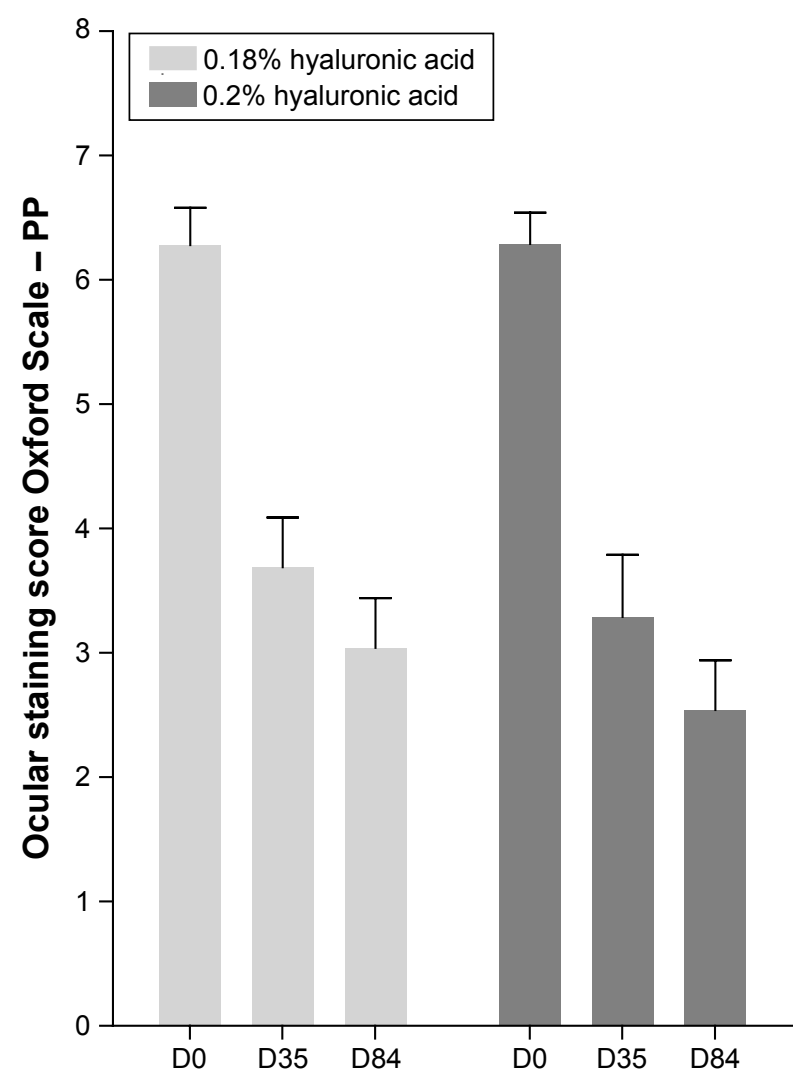

Figure I Composite staining score of cornea and conjunctiva (Oxford Scale, mean \pm SEM) at D0, D35, and D84.

Abbreviations: SEM, standard error of mean; D, days; PP, per-protocol.

increased from -3.00 (D35) to -4.06 (D84) with $0.2 \% \mathrm{HA}$ and from -2.59 (D35) to -3.54 (D84) with $0.18 \%$ HA. The difference between the medications observed at D35 is more pronounced at D84 in favor of $0.2 \%$ HA treatment (Figure 1).

All symptoms of the 6-item OCI improved during the study in frequency and intensity (Figure 2). For each of the 12 items, the amplitude of improvement in frequency and intensity tended to be constantly higher with $0.2 \%$ HA compared to $0.18 \%$ HA. There was no statistically significant difference between treatments, except for the symptom "pain," which was significantly lower with $0.2 \%$ HA at D84, compared to $0.18 \%$ HA (Mann-Whitney $U$-test $U=2.085, P<0.0370)$. At D84, all OCI items were reduced by $\sim 40 \%-50 \%$, compared to D0.

TBUT improved slightly with both treatments over time. At D84, TBUT had increased by $4.7 \pm 6.2 \mathrm{~s}$ in the $0.2 \%$ HA group and by $5.4 \pm 8.6 \mathrm{~s}$ in the $0.18 \%$ HA group (sum of 3 measurements), compared to baseline values at D0.

As secondary analysis, the overall score of the 15-point Oxford Scale primary endpoint was applied on the ITT population $(n=83)$ at D35. This analysis confirmed the noninferiority of $0.2 \% \mathrm{HA}$ vs $0.18 \% \mathrm{HA}$.
All other analysis on secondary endpoints performed at D35 and D84 on the PP population demonstrated similar results with no significant statistical difference between the two treatments. This included the overall score of the Oxford Scale (Figure 1), subscores of the corneal, temporal and nasal zones, TBUT change on D35 (sum of 3 measures: $0.2 \%$ HA: $+3.21 \mathrm{~s} \pm 5.67 ; 0.18 \% \mathrm{HA}:+2.41 \mathrm{~s} \pm 5.63)$, and the 12-item OCI on symptoms of dryness in frequency and intensity, except significant difference for item "pain" (Figure 2C) and patients' and investigators' evaluation of treatment (Table 4).

\section{Adverse events}

Five adverse events (AEs) were reported in 4 patients during the study: $1 \mathrm{AE}$ (episodic allergy) in 1 patient out of $43(2.3 \%)$ in the $0.2 \%$ HA group and 4 AEs ( 1 patient: grittiness exacerbation, 1 patient: ocular irritation, 1 patient: important ocular fatigue + ocular irritation) in 3 patients out of $42(7.1 \%)$ in the $0.18 \%$ HA group. Two patients in the $0.18 \%$ HA group discontinued treatment due to the occurrence of an $\mathrm{AE}$, and 1 patient in the $0.2 \%$ HA group. All AEs resolved without sequelae. No serious AE was reported.

\section{Patient preference}

Eye drops of $0.2 \% \mathrm{HA}$ and $0.18 \%$ HA received similar assessments by the investigators and patients. Scores for efficacy (investigators) and satisfaction (patients) were all close to 5 (maximum of 7) (Table 4).

\section{Discussion}

This study found significantly lowered corneal and conjunctival staining values of the Oxford Scale after therapy with $0.2 \%$ and $0.18 \%$ HA eye drops. Moreover, the 6-item OCI decreased in frequency and intensity during the study. TBUT values increased from D0 to D84 by $1.67 \mathrm{~s}$ in the $0.2 \% \mathrm{HA}$ group and by $1.8 \mathrm{~s}$ in the $0.18 \% \mathrm{HA}$ group. This small, but significant, increase in tear stability is in the order of magnitude reported in a recent meta-analysis on tear substitutes by Doughty. ${ }^{21}$

Our results suggest that OS improved during HA treatment, which is in accordance with other clinical findings. Shimmura et $\mathrm{al}^{7}$ were the first to use two dyes for OS staining (fluorescein and rose bengal) as efficacy markers for clinical effects of HA eye drops. Preservative-free $0.1 \%$ HA was applied six times daily for 4 weeks. The investigators found that corneal fluorescein staining decreased, while no change in rose bengal staining was noted. A concentration of $0.15 \%$ HA was used by Brjesky et al. ${ }^{22}$ Among positive 

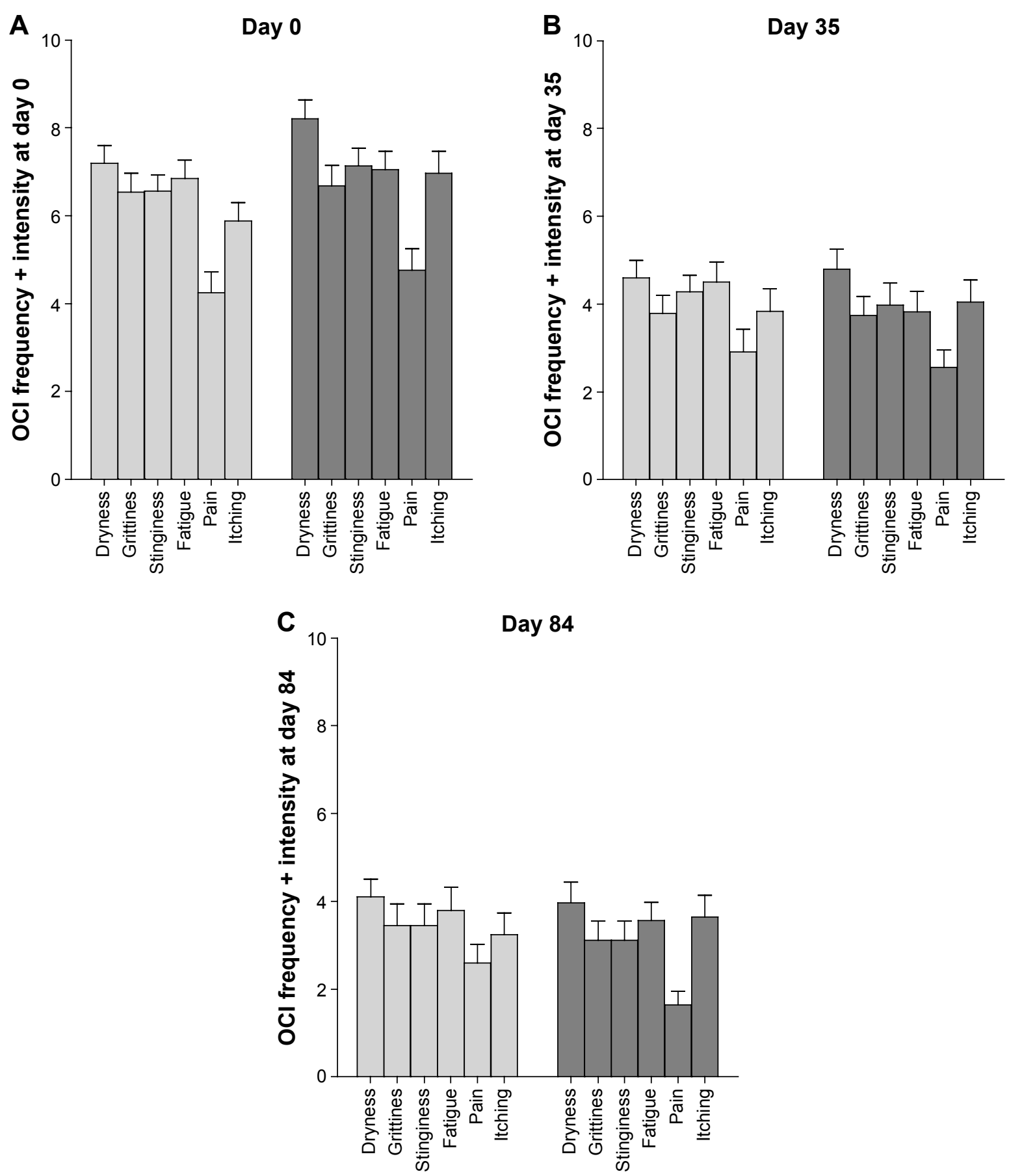

$\square 0.18 \% \mathrm{HA} \quad \square 0.2 \% \mathrm{HA}$

Figure $2 \mathrm{OCl}$ as sum of frequency and intensity of 6 items (mean \pm SEM) on Day $0(\mathbf{A})$, Day 35 (B), and Day 84 (C).

Notes: At Day 84 , item "pain" was significantly more reduced by $0.2 \%$ HA than by $0.18 \%$ HA $(P<0.0370)$. All other differences between treatments were not significant. Abbreviations: $\mathrm{HA}$, hyaluronic acid; $\mathrm{OCl}$, ocular comfort index; SEM, standard error of mean.

results for TBUT and Schirmer test, they observed decreased staining values. However, there is no information on the type of the dye the investigators used. Pinto-Fraga et $\mathrm{al}^{14}$ and Lopez-de la Rosa et $\mathrm{al}^{13}$ describe in double-masked, randomized trials reduced corneal and conjunctival staining after 1 month application of unpreserved $0.2 \%$ or
$0.3 \%$ HA eye drops, respectively, while saline $0.9 \%$ alone yielded increased staining values, indicating exacerbation of DED. In another study, $0.4 \%$ HA was used and led to a decrease in rose bengal staining after 7 days of treatment, probably due to the higher HA concentration. ${ }^{8}$ It may be concluded that $0.1 \%$ HA in eye drops may not be adequate 
Table 4 Efficacy according to the investigator and satisfaction assessed by the patient

\begin{tabular}{lccccccc}
\hline Group & $\mathbf{N}$ & Nmiss & Mean & SD & Min & Max & Median \\
\hline D84: efficacy (investigators) & & & & \\
0.2\% HA & 36 & 0 & 4.83 & 1.46 & 2.0 & 7.0 & 5.0 \\
0.18\% HA & 29 & 0 & 5.07 & 1.53 & 2.0 & 7.0 & 5.0 \\
D84: satisfaction (patients) & & & & \\
0.2\% HA & 36 & 0 & 4.86 & 1.50 & 1.0 & 7.0 & 5.0 \\
0.18\% HA & 29 & 0 & 5.07 & 1.58 & 2.0 & 7.0 & 5.0 \\
\hline
\end{tabular}

Notes: 7-point Lickert scale with $7=$ very satisfied and $0=$ not at all satisfied.

Abbreviations: $\mathrm{HA}$, hyaluronic acid; SD, standard deviation; D, day; Min, minimum; Max, maximum; Nmiss, missing number.

to improve rose bengal staining in DED but enough for fluorescein staining.

In the present study, OS staining served as the primary outcome variable. On D35, a 47.7\% reduction in staining score in the $0.2 \%$ HA treatment group and a $41.2 \%$ reduction in patients treated with $0.18 \%$ HA were observed. Staining values further decreased between D35 and D84. On D84, staining was reduced $64.5 \%$ with $0.2 \% \mathrm{HA}$ and $56.4 \%$ with $0.18 \%$ HA, compared to baseline values at D0. Despite the tendency for lesser staining with $0.2 \%$ HA eye drops, there was no statistical difference in our study between $0.2 \%$ and $0.18 \%$ HA. Statistical analysis of the present data, based on criteria predefined in the study protocol, shows noninferiority of $0.2 \% \mathrm{HA}$ compared to $0.18 \% \mathrm{HA}$. Investigators and patients assessed both eye drops with a median score of 5 of 7 points (Likert scale).

Alteration of the OS can be considered a hallmark of DED and thus an important clinical marker for therapy success. ${ }^{23}$ HA has excellent moisturization and surface cell adhesion properties and is a component of the natural tear fluid. ${ }^{24}$ Its properties led to the development of HA-containing eye drops and gels for DED treatment. HA eye drops may be promoting OS tissue healing by humidifying the OS, thus bringing back the integrity of corneal and conjunctival epithelium. It has been shown that topically applied HA stimulates wound healing in an animal model ${ }^{25}$ and attenuates oxidative stress and inflammation, thus improving dry eye symptoms. ${ }^{26}$

Both eye drops of this study were unpreserved but had different excipients ( $0.2 \%$ HA: sorbitol, citrate buffer, $0.18 \%$ HA: sodium chloride, potassium, magnesium, calcium, disodium hydrogen phosphate, sodium citrate). While it cannot be excluded that excipients could account for the slightly higher efficacy of $0.2 \% \mathrm{HA}$, the difference could be due to the $0.02 \%$ higher HA concentration, compared to $0.18 \%$. As Abelson ${ }^{27}$ points out, direct comparisons of HA products should take into account that the viscoelasticity and efficacy of HA-based products do vary not only by concentration but also depend on molecular weight.

The study safety data were consistent with the known safety profile of both tested HA eye drops and were judged as being related to the dry eye underlying condition. The rate of AEs and treatment discontinuation due to AEs was $2.3 \%$ for $0.2 \%$ HA and $7.1 \%$ for $0.18 \%$ HA. No serious AE was reported.

Both eye drops caused clinically and statistically relevant improvement of $>50 \%$ in staining score and $40 \%-50 \%$ in OCI throughout the study. The data suggest that $0.2 \%$ $\mathrm{HA}$ and $0.18 \%$ HA are efficacious concentrations of HA for treatment of moderate to severe dry eye. Safety data were consistent with the known safety profile of both investigational products. Thus, $0.2 \% \mathrm{HA}$ and $0.18 \% \mathrm{HA}$ fit well into the big portfolio of novel topical treatment options of DED. ${ }^{28}$

\section{Conclusion}

Our study suggests that both $0.2 \% \mathrm{HA}$ and $0.18 \% \mathrm{HA}$ are efficacious and safe for the treatment of moderate to severe DED.

\section{Acknowledgments}

This clinical study was funded by Ursapharm. The authors thank Klaus Rudolph of Rudolph Consulting for writing and editorial support, funded by Ursapharm.

The authors thank the French study centers involved in this study (details available on request).

\section{Disclosure}

Groß is an employee of Ursapharm. Childs is the CEO of Laboratoires Delbert, which was appointed as Clinical Research Organization for the clinical study by Ursapharm. The authors report no other conflicts of interest in this work.

\section{References}

1. The definition and classification of dry eye disease: report of the Definition and Classification Subcommittee of the International Dry Eye WorkShop (2007). Ocul Surf. 2007;5(2):75-92.

2. Management and therapy of dry eye disease: report of the Management and Therapy Subcommittee of the International Dry Eye WorkShop (2007). Ocul Surf. 2007;5(2):163-178.

3. Steven $P$, Cursiefen C. [Anti-inflammatory treatment in dry eye disease]. Klin Monbl Augenheilkd. 2012;229(5):500-505. German.

4. van de Wouw DS, van der Meulen IJ, van Vliet JM, Lapid-Gortzak R, Nieuwendaal CP, van den Berg TJ. Increased straylight in patients with keratoconjunctivitis Sicca. Cornea. 2016;35(6):749-753.

5. The epidemiology of dry eye disease: report of the Epidemiology Subcommittee of the International Dry Eye WorkShop (2007). Ocul Surf. 2007;5(2):93-107.

6. Klingenberg HO. Excipient update - a real eyeopener: advances in hyaluronic acid for ophthalmology. Drug Dev Deliver. 2013;13(4). 
7. Shimmura S, Ono M, Shinozaki K, et al. Sodium hyaluronate eyedrops in the treatment of dry eyes. Br J Ophthalmol. 1995;79(11):1007-1011.

8. Troiano P, Monaco G. Effect of hypotonic $0.4 \%$ hyaluronic acid drops in dry eye patients: a cross-over study. Cornea. 2008;27(10): 1126-1130.

9. Liu XM, Harmon PS, Maziarz EP, Rah MJ, Merchea MM. Comparative studies of hyaluronan in marketed ophthalmic products. Optom Vis Sci. 2014;91(1):32-38.

10. Baudouin C, Cochener B, Pisella PJ, et al. Randomized, phase III study comparing osmoprotective carboxymethylcellulose with sodium hyaluronate in dry eye disease. Eur J Ophthalmol. 2012;22(5): 751-761.

11. Baeyens V, Bron A, Baudouin C; Vismed/Hylovis Study G. Efficacy of $0.18 \%$ hypotonic sodium hyaluronate ophthalmic solution in the treatment of signs and symptoms of dry eye disease. J Fr Ophtalmol. 2012;35(6):412-419.

12. Lee JH, Ahn HS, Kim EK, Kim TI. Efficacy of sodium hyaluronate and carboxymethylcellulose in treating mild to moderate dry eye disease. Cornea. 2011;30(2):175-179.

13. Lopez-de la Rosa A, Pinto-Fraga J, Blazquez Arauzo F, Urbano Rodriguez R, Gonzalez-Garcia MJ. Safety and efficacy of an artificial tear containing $0.3 \%$ hyaluronic acid in the management of moderateto-severe dry eye disease. Eye Contact Lens. Epub 2016 May 26.

14. Pinto-Fraga J, Lopez-de la Rosa A, Blazquez Arauzo F, Urbano Rodriguez R, Gonzalez-Garcia MJ. Efficacy and safety of $0.2 \%$ hyaluronic acid in the management of dry eye disease. Eye Contact Lens. 2017;43(1):57-63.

15. Teping C. Hyaluronsäure. Thieme Drug Report. 2010;2(3):1-12.

16. De Saint Jean M, Debbasch C, Brignole F, Warnet JM, Baudouin C. Relationship between in vitro toxicity of benzalkonium chloride (BAC) and preservative-induced dry eye. Adv Exp Med Biol. 2002; 506(Pt A):697-702.

17. Bron AJ, Evans VE, Smith JA. Grading of corneal and conjunctival staining in the context of other dry eye tests. Cornea. 2003;22(7): $640-650$.
18. Romanchuk KG. Fluorescein. Physiochemical factors affecting its fluorescence. Surv Ophthalmol. 1982;26(5):269-283.

19. Kim J, Foulks GN. Evaluation of the effect of lissamine green and rose bengal on human corneal epithelial cells. Cornea. 1999;18(3): 328-332.

20. McLeod SA. Likert Scale. [homepage on the Internet]; 2008. Available from: www.simplypsychology.org/likert-scale.html. Accessed September 29, 2016.

21. Doughty MJ. Fluorescein-tear breakup time as an assessment of efficacy of tear replacement therapy in dry eye patients: a systematic review and meta-analysis. Ocul Surf. 2014;12(2):100-111.

22. Brjesky VV, Maychuk YF, Petrayevsky AV, Nagorsky PG. Use of preservative-free hyaluronic acid $\left(\mathrm{Hylabak}^{\circledR}\right)$ for a range of patients with dry eye syndrome: experience in Russia. Clin Ophthalmol. 2014;8: 1169-1177.

23. Foulks GN. Challenges and pitfalls in clinical trials of treatments for dry eye. Ocul Surf. 2003;1(1):20-30.

24. Dreyfuss JL, Regatieri CV, Coelho B, et al. Altered hyaluronic acid content in tear fluid of patients with adenoviral conjunctivitis. An Acad Bras Cienc. 2015;87(1):455-462.

25. Ho WT, Chiang TH, Chang SW, Chen YH, Hu FR, Wang IJ. Enhanced corneal wound healing with hyaluronic acid and high-potassium artificial tears. Clin Exp Optom. 2013;96(6):536-541.

26. Macri A, Scanarotti C, Bassi AM, et al. Evaluation of oxidative stress levels in the conjunctival epithelium of patients with or without dry eye, and dry eye patients treated with preservative-free hyaluronic acid $0.15 \%$ and vitamin B12 eye drops. Graefes Arch Clin Exp Ophthalmol. $2015 ; 253(3): 425-430$.

27. Abelson MB, Lafon A. 3,500 years of artificial tears. Rev Ophthalmol. 2014;14(12):44-47.

28. Nebbioso M, Fameli V, Gharbiya M, Sacchetti M, Zicari AM, Lambiase A. Investigational drugs in dry eye disease. Expert Opin Investig Drugs. 2016;25(12):1437-1446.
Clinical Ophthalmology

\section{Publish your work in this journal}

Clinical Ophthalmology is an international, peer-reviewed journal covering all subspecialties within ophthalmology. Key topics include: Optometry; Visual science; Pharmacology and drug therapy in eye diseases; Basic Sciences; Primary and Secondary eye care; Patient Safety and Quality of Care Improvements. This journal is indexed on Submit your manuscript here: http://www.dovepress.com/clinical-ophthalmology-journal

\section{Dovepress}

PubMed Central and CAS, and is the official journal of The Society of Clinical Ophthalmology (SCO). The manuscript management system is completely online and includes a very quick and fair peer-review system, which is all easy to use. Visit http://www.dovepress.com/ testimonials.php to read real quotes from published authors. 